\title{
Proper motion studies of outflows from Classical T Tauri stars
}

\author{
F. McGroarty ${ }^{1,2}$, T. P. Ray ${ }^{2}$, and D. Froebrich ${ }^{3}$
}

\author{
1 Department of Experimental Physics, National University of Ireland Maynooth, Maynooth, Co. Kildare, Ireland \\ e-mail: fiona.mcgroarty@nuim.ie \\ 2 Dublin Institute for Advanced Studies, 5 Merrion Square, Dublin 2, Ireland \\ ${ }^{3}$ Centre for Astrophysics \& Planetary Science, School of Physical Sciences, University of Kent, Canterbury CT2 7NH, UK
}

Received 4 December 2006 / Accepted 7 March 2007

\begin{abstract}
In a previous paper (McGroarty \& Ray 2004) we examined the environment of a number of evolved low-mass young stars, i.e. Classical T Tauri Stars, to see if they are capable of driving parsec-scale outflows. These stars - CW Tau, DG Tau, DO Tau, HV Tau C and RW Aur - were previously known to drive only "micro-jets" or small-scale outflows of $\leq 1$ ' or 0.04 pc at the distance of the Taurus-Auriga Cloud. We found that they drive outflows of $0.5 \mathrm{pc}-1 \mathrm{pc}$, based on the morphology and alignment of newly discovered and previously known $\mathrm{HH}$ objects with these sources and their "micro-jets". Here, we use a cross-correlation method to determine the proper motions of the HH objects in these five outflows (HH 220, HH 229, HH 702, HH 705 and HH 826 - HH 835) which in turn allows us to confirm their driving sources. Moreover, the tangential velocities of $\mathrm{HH}$ objects at large distances from their origin are currently poorly known so these proper motions will allow us to determine how velocities evolve with distance from their source. We find tangential velocities of typically $200 \mathrm{~km} \mathrm{~s}^{-1}$ for the more distant objects in these outflows. Surprisingly, we find similar tangential velocities for the "micro-jets" that are currently being ejected from these sources. This leads us to suggest that either the outflow velocity was much higher $10^{3}$ years ago when the more distant objects were ejected and that these objects have decelerated to their current velocity or that the outflow velocity at the source has remained approximately constant and the more distant objects have not undergone significant deceleration due to interactions with the ambient medium. Numerical simulations are needed before we can decide between these scenarios.
\end{abstract}

Key words. ISM: Herbig-Haro objects - ISM: jets and outflows - stars: pre-main sequence - stars: formation - stars: general

\section{Introduction}

Herbig-Haro $(\mathrm{HH})$ objects are the optical emission line tracers of outflows from young stars. Although the exact mechanism by which outflows are ejected from a young forming star are still unclear, it is accepted that they are intrinsically linked to accretion (Cabrit et al. 1990; Hartigan et al. 1995). Close to the source these $\mathrm{HH}$ objects are usually seen as well collimated jets, however further out they separate into discrete knots or diffuse regions, in some cases taking the form of a bow shock - the objects in the HH34 outflow is a typical example of such as structure (Devine et al. 1997). Initially these HH outflows were assumed to be a fraction of a parsec in length, with only a few exceptions (e.g. Ray 1987). However the past decade has revealed many parsec-scale outflows (Bally \& Devine 1997; Reipurth \& Bally 2001), which are often comparable in size to their parent cloud.

The majority of outflows are found to be driven by low-mass young stars, which can be classified by the amount of excess infrared emission in their spectral energy distribution. This excess is due to circumstellar dust and is very strong in the young, embedded Class I sources and is almost non-existent in the most evolved Class III sources (Lada \& Wilking 1984; Lada 1987). Parsec-scale optical outflows are generally found to be driven by low-mass Class I sources, however a number of intermediatemass sources have recently been observed to drive large-scale outflows (McGroarty et al. 2004).
In McGroarty \& Ray (2004) (hereafter referred to as MR04) we examined a number of more evolved, Class II low-mass sources (i.e. Classical T Tauri Stars - CTTSs) and found they also drive large-scale outflows. The sources observed in MR04 (CW Tau, DG Tau, DO Tau, HV Tau C and RW Aur) were previously only known to drive "micro-jets" or small-scale outflows of $\leq 1^{\prime}$. This corresponds to $0.04 \mathrm{pc}$ at the distance $(140 \mathrm{pc})$ to the Taurus-Auriga Cloud (Elias 1978; Wichmann et al. 1998). Our observations in MR04 show them to actually drive outflows of $\sim 0.5-1$ pc. Although these outflows are not as large, or as spectacular, as those from younger sources, they are less embedded so are more easily observed.

Hartigan et al. (1995) have shown that mass outflow rates are directly correlated with mass accretion rates. The stellar envelope dissipates over time as material is accreted onto the protostar thus accretion rates should decrease with time (Ward-Thompson 2002). Bontemps et al. (1996) show observational evidence for this with a decline in molecular outflow strength from Class 0 to Class I Young Stellar Objects (YSOs). We can infer a further decline in Class II YSOs. Despite this CTTSs are still capable of driving collimated atomic/ionized large-scale outflows (MR04). We see that their morphologies are similar to those from Class I sources, but how do the lower accretion rates affect their kinematics? Lower accretion rates translate into lower mass loss rates and hence a decrease in the mechanical luminosity. However, as the average ambient density is lower in a Class II environment the outflow velocity may not be reduced 
much by interaction with its surroundings. Determining velocities for the distant $\mathrm{HH}$ objects in these CTTS outflows is thus fundamental to discovering the effect that the lower accretion rates of CTTSs and the lower parent cloud density will have on outflows driven by such sources.

As a continuation of the MR04 study, here we use multiepoch observations to determine proper motions and hence tangential velocities for the $\mathrm{HH}$ objects previously discovered from our CTTS sources. This will allow us to test whether the driving sources determined in MR04, largely based on outflow morphology and alignment, are correct. Additionally, the tangential velocities of $\mathrm{HH}$ objects at relatively large distances from their source are poorly known. In particular, how these velocities evolve with distance from the source is not well understood. Both of these issues are addressed here in light of the results of the proper motion studies.

Dynamical timescales were estimated for these outflows in MR04 based on the assumption that the most distant objects are moving with a tangential velocity of $50 \mathrm{~km} \mathrm{~s}^{-1}$, which is a lower limit for the velocity needed to induce optically visible shocks. This is a conservative estimate and the more accurate tangential velocities obtained will allow more realistic dynamical timescales to be calculated. In any event, however, it is important to note that these timescales are much less than the age of the outflow as estimated from evolutionary tracks, and only represent their optically visible portion. There is little doubt that these outflows have blown out of their parent cloud (MR04).

Details about the observations and proper motion method used are given in Sects. 2 and 3 respectively. The results of this study are reported in Sect. 4 and the implications of these results are discussed in Sect. 5 with our conclusions being presented in Sect. 6.

\section{Observations}

Using multi-epoch observations the velocity and direction of motion are determined for many of the known $\mathrm{HH}$ objects in the vicinity of CW Tau, DG Tau, DO Tau, HV Tau C and RW Aur. The observations used here were taken on a number of different observing runs using the Wide Field Camera on the Isaac Newton Telescope, La Palma (Canary Islands). The same CCDs and hence the same angular resolution is used for all observations - 1 pixel projects to 0.33 square on the sky. The observing runs occurred in November 1999 (epoch 1999.91), February 2001 (epoch 2001.12), December 2002 (epoch 2002.98) and finally November 2003 (epoch 2003.91), however not all regions were targeted during each run. Narrow band filters were used to observe the $\mathrm{HH}$ objects, both $[\mathrm{SII}]\left(\lambda_{\mathrm{c}}=672.5 \mathrm{~nm}, \Delta \lambda(F W H M)=\right.$ $8.0 \mathrm{~nm})$ and $\mathrm{H} \alpha\left(\lambda_{\mathrm{c}}=656.8 \mathrm{~nm}, \Delta \lambda(F W H M)=9.5 \mathrm{~nm}\right)$ are used here, but again not all regions were observed using both of these filters in each run. Table 1 lists the observations for each region. Exposure times for the narrow band images were typically $30 \mathrm{~min}$. The data was reduced using standard IRAF procedures for bias subtraction and flat fielding.

\section{Proper motion method}

A cross-correlation method was devised to determine the proper motions of the $\mathrm{HH}$ objects in the vicinity of our five CTTS sources. The images from two different epochs are initially aligned using star positions obtained from the SExtractor software (Bertin \& Arnouts 1996). Our method then maps one of
Table 1. Log of observations used for each source in the proper motions study.

\begin{tabular}{llccc}
\hline \hline Region & Line & 1st Epoch & 2nd Epoch & 3rd Epoch \\
\hline DG Tau & {$[\mathrm{SII}]$} & 1999.91 & 2001.12 & 2003.91 \\
& $\mathrm{H} \alpha$ & 2001.12 & 2003.91 & \\
CW Tau & {$[\mathrm{SII}]$} & 2001.12 & 2002.98 & 2003.91 \\
& $\mathrm{H} \alpha$ & 2001.12 & 2002.98 & 2003.91 \\
DO Tau & {$[\mathrm{SII}]$} & 2001.12 & 2002.98 & 2003.91 \\
& $\mathrm{H} \alpha$ & 2001.12 & 2002.98 & 2003.91 \\
HV Tau C & {$[\mathrm{SII}]$} & 2001.12 & 2002.98 & 2003.91 \\
& $\mathrm{H} \alpha$ & 2001.12 & 2002.98 & 2003.91 \\
RW Aur & {$[\mathrm{SII}]$} & 2001.12 & 2003.91 & \\
& $\mathrm{H} \alpha$ & 2001.12 & 2003.91 & \\
\hline
\end{tabular}

the images onto the other using a polynomial fit (up to third order) of the star positions and the IRAF task geomap. This procedure ensures the correction of any systematic effects such as stretching, rotation, etc. of the image.

The smallest time difference between epochs in Table 1 is 0.93 years (epoch 2003.91-epoch 2002.98). A bright, compact $\mathrm{HH}$ knot that has a tangential velocity of $50 \mathrm{~km} \mathrm{~s}^{-1}$ at a distance of 140 pc will appear to shift 0.21 pixels with respect to the background stars in this time. Our cross correlation method samples to a spatial frequency of 0.1 pixels, so even such low velocities could, in principle, be detected.

However, there are two main sources of error in these measurements. The first is due to the accuracy of the alignment of the images. This is typically $0.16-0.4$ pixels (i.e. $\pm(0.08-0.2)$ pixels $)$ in both $x$ and $y$ directions, generating errors of about $18-56 \mathrm{~km} \mathrm{~s}^{-1}$ or $\pm(9-28) \mathrm{km} \mathrm{s}^{-1}$. This is determined by using all combinations of epochs listed in Table 1, so is valid for all epoch separation timescales. All errors in velocity and direction are quoted at $1 \sigma$. However the errors in the central frame of the CW Tau region (i.e. HH 826, HH 220 NW and HH 828, see Fig. 1) are much higher than this. There are very few stars in this field and consequently the alignment is poor. Alignment errors for these objects are about 1 pixel in $x$ and $y$ i.e. $\sim 110 \mathrm{~km} \mathrm{~s}^{-1}\left( \pm \sim 55 \mathrm{~km} \mathrm{~s}^{-1}\right)$. The second source of error is in the position of the $\mathrm{HH}$ object and is due to its signal-to-noise ratio, PSF (point spread function) and extent. This seems to be the limiting error for most of the objects observed here, especially the faint objects as one would expect. Typically these errors are about $0.2-0.5$ pixels (i.e. $\pm(0.1-0.25)$ pixels) in $x$ and $y$, corresponding to $\sim 20-55 \mathrm{~km} \mathrm{~s}^{-1}$ or $\pm(10-28) \mathrm{km} \mathrm{s}^{-1}$. The most noticeable exception are the very faint $\mathrm{HH} 830$ knots (in the vicinity of DG Tau) which in [SII] have errors of about 1.1 pixels, i.e. $\sim 120 \mathrm{~km} \mathrm{~s}^{-1}\left( \pm \sim 60 \mathrm{~km} \mathrm{~s}^{-1}\right)$ and in $\mathrm{H} \alpha$ have errors of $\sim 0.7$ pixels in $x$ and $y$ i.e. $\sim 80 \mathrm{~km} \mathrm{~s}^{-1}\left( \pm \sim 40 \mathrm{~km} \mathrm{~s}^{-1}\right)$. The errors in velocity for each frame/image as explained above are given in the caption for each table where results are stated. The errors in direction of motion are given separately for each object, as the error in direction depends on the velocity - see Tables 3 to 6 .

A common problem with proper motion measurements can be that only the global motion of a $\mathrm{HH}$ object is obtained. Here, however, we have managed to get proper motions for individual knots in some of the larger HH objects, e.g. HH 827, HH 702, HH 831 and HH705. In some cases it is advantageous to look at the global motion of a $\mathrm{HH}$ complex to ascertain a possible driving source and then to examine the individual motions within it. This has been done for HH 827 (Sect. 4.1), HH 702 (Sect. 4.2) and HH 705 and HH 831 (Sect. 4.3).

One of the main causes of false proper motions is photometric variability. Occasionally, the majority of knots in a large 

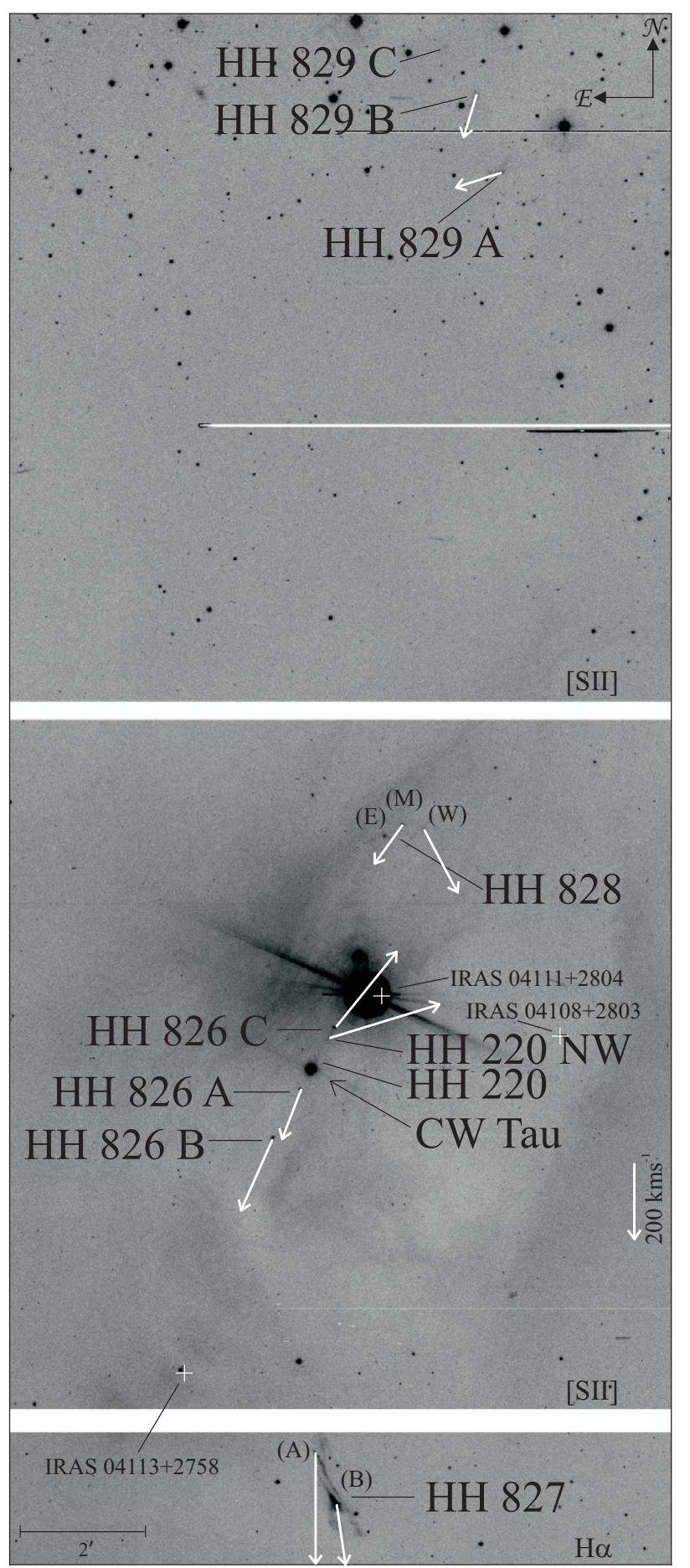

Fig. 1. Proper motions of HH $826-\mathrm{HH} 829$ in the vicinity of CW Tau. The direction of motion of each $\mathrm{HH}$ object is represented by white arrows. The relative length of these arrows denotes the relative velocity of the object. The redshifted (HH 220) jet is also marked above, however it is too faint for proper motion studies. These vectors show that $\mathrm{HH} 826$ and $\mathrm{HH} 827$ are driven by CW Tau, assuming precession of the outflow over time. However this study also shows that HH 828 and HH 829 are not driven by CW Tau as previously suggested. Candidate sources for these objects are suggested in the text.

HH complex will be moving in one direction, while an individual knot may appear to move in a completely different direction. In this case, the different motion may be assigned to a change in relative brightness within that knot rather than physical
Table 2. Details of the HH objects found in our recent study (MR04) of extended outflows from CTTSs. Angular separation and position angle (PA) are with respect to the suggested source. The results of proper motion studies of these HH objects are given in Sect. 4.

\begin{tabular}{|c|c|c|c|}
\hline Object & $\begin{array}{l}\text { Suggested } \\
\text { Source }^{a}\end{array}$ & $\begin{array}{l}\text { Angular } \\
\text { Separation }\end{array}$ & $\mathrm{PA}$ \\
\hline HH 826 A & CW Tau & 0,37 & $153^{\circ}$ \\
\hline HH $826 \mathrm{~B}$ & CW Tau & 1.27 & $153^{\circ}$ \\
\hline HН 827 & CW Tau & 6.2 & $184^{\circ}$ \\
\hline HH $826 \mathrm{C}$ & CW Tau & 0.77 & $326^{\circ}$ \\
\hline HH $828 \mathrm{E}$ & $?^{b}$ & & \\
\hline HH $828 \mathrm{M}$ & $?^{b}$ & & \\
\hline HH $828 \mathrm{~W}$ & $?^{b}$ & & \\
\hline HH 829 A & CW Tau & $14^{\prime} .9$ & $348^{\circ}$ \\
\hline HH 829 B & CW Tau & $166^{\prime} 12$ & $351^{\circ}$ \\
\hline $\mathrm{HH} 829 \mathrm{C}$ & CW Tau & 16.8 & $353^{\circ}$ \\
\hline $\mathrm{HH} 830 \mathrm{~A}$ & DG Tau & $9 \cdot 6$ & $48^{\circ}$ \\
\hline HH 830 B & DG Tau & $11: 3$ & $48^{\circ}$ \\
\hline $\mathrm{HH} 830 \mathrm{C}$ & DG Tau & $14 ! 3$ & $48^{\circ}$ \\
\hline HН $831 \mathrm{~A}$ & DO Tau & $10 ! 8$ & $74^{\circ}$ \\
\hline HН 831 B & DO Tau & $11^{\prime}$ & $74^{\circ}$ \\
\hline НH 832 & DO Tau & $7: 7$ & $78^{\circ}$ \\
\hline HH 833 & HV Tau C & 4.6 & $25^{\circ}$ \\
\hline HH 834 & $?^{c}$ & & \\
\hline HH 835 & RW Aur & 5.37 & $310^{\circ}$ \\
\hline
\end{tabular}

${ }^{a}$ Suggested source as given in MR04.

${ }^{b}$ While the HH828 knots are aligned with the extended CW Tau outflow we suggest in MR04 that, based on their morphology, they are not driven by CW Tau.

${ }^{c} \mathrm{HH} 834$ is in the DO Tau/HV Tau C region however no candidate driving sources could be suggested in MR04.

motion. That said, the cooling time is typically much longer than the timespan between our measurements so such false measurements are usually not a problem.

\section{Results}

A detailed introduction to the CTTSs DG Tau, CW Tau, DO Tau, HV Tau C and RW Aur and their associated "micro-jets"/smallscale outflows is given in MR04. The newly discovered objects (HH 826 - HH 835, see Table 2) that may be extensions to these outflows are also presented there. Here, a brief summary is given for each outflow before the proper motion results are discussed.

\subsection{CW Tau}

The HH 220 bipolar "micro-jet" from CW Tau was first discovered by Gomez de Castro (1993). Hirth et al. (1994) show this outflow to be $4^{\prime \prime}-6^{\prime \prime}$ on either side of CW Tau. In MR04 we presented a number of $\mathrm{HH}$ objects which appear to be an extension to this outflow. HH $826 \mathrm{~A}, \mathrm{HH} 826 \mathrm{~B}$ and $\mathrm{HH} 827$ are to the south of CW Tau, with HH $826 \mathrm{C}$, HH 828 and HH 829 to the north (see Fig. 1). In MR04 we assumed precession of the outflow over time, which gives the outflow an elongated inverted "S" shape i.e. that it consisted of HH 826, HH 827 and HH829. This suggested a total projected length for the outflow of $\sim 1$ pc. The source of the HH 828 trio of knots was unclear. Their alignment with the HH220 jet suggests that they are part of the CW Tau outflow, however the spatial spread of the knots could only occur if the outflow became much less collimated between $\mathrm{HH} 826 \mathrm{C}$ and $\mathrm{HH} 828$. As the southern outflow from CW Tau remains collimated over a larger distance (to HH 827) it is probable that $\mathrm{HH} 828$ is not driven by CW Tau. 
Table 3. Tangential velocity and direction of motion of HH220, HH 826, HH 827, HH 828 and HH 829 in the vicinity of CW Tau assuming a distance of $140 \mathrm{pc}$ to the source (Elias 1978; Wichmann et al. 1998). The associated errors for HH 826, HH $220 \mathrm{NW}$ and $\mathrm{HH} 828$ are $\pm \sim 55 \mathrm{~km} \mathrm{~s}{ }^{-1}$. For HH 827 and HH 829 the errors are $\pm(10-28) \mathrm{km} \mathrm{s}^{-1}$ (see Sect. 3). All errors are 1 $\sigma$. For Tables 3-6 the discrepancies between the [SII] and $\mathrm{H} \alpha$ measurements are discussed in the text and in Sect. 5.3.

\begin{tabular}{|c|c|c|c|c|c|c|}
\hline \multirow[b]{2}{*}{ HH Object } & \multirow[b]{2}{*}{ Source $^{a}$} & \multirow[b]{2}{*}{$\begin{array}{l}\text { Ang. } \\
\text { Sep. }\end{array}$} & \multicolumn{2}{|c|}{ [SII] } & \multirow{2}{*}{\multicolumn{2}{|c|}{$\begin{array}{l}\mathrm{H} \alpha \\
\text { Direction } \\
/^{\circ}\end{array}$}} \\
\hline & & & $\begin{array}{l}\text { Velocity } \\
/ \mathrm{km} \mathrm{s}^{-1}\end{array}$ & $\begin{array}{l}\text { Direction } \\
/^{\circ}\end{array}$ & & \\
\hline HH 826 A & CW Tau (B) & 0,4 & 132 & $159 \pm 24$ & & \\
\hline HH 826 B & CW Tau (B) & 1.2 & 207 & $156 \pm 15$ & 282 & $166 \pm 11$ \\
\hline HH 827 A & CW Tau (B) & 6.1 & & & 347 & $180 \pm(2-5)$ \\
\hline HH 827 B & CW Tau (B) & 6.9 & 199 & $198 \pm(3-8)$ & 151 & $188 \pm(4-11)$ \\
\hline HH 220 NW & CW Tau (R) & 0.6 & 323 & $287 \pm 10$ & & \\
\hline HH 826 C & CW Tau (R) & 0.8 & 261 & $321 \pm 12$ & 111 & $26 \pm 29$ \\
\hline HH $828 \mathrm{M}$ & & & 118 & $144 \pm 27$ & & \\
\hline HH $828 \mathrm{~W}$ & & & 173 & $208 \pm 18$ & & \\
\hline HH 829 A & & & 116 & $108 \pm(5-14)$ & & \\
\hline HH 829 B & & & 113 & $163 \pm(5-14)$ & & \\
\hline
\end{tabular}

${ }^{a}$ For the $\mathrm{HH}$ objects that are driven by $\mathrm{CW}$ Tau, B and R denotes whether the object is aligned with the blue or redshifted lobe.

Using our cross-correlation method we measure the proper motions of HH 826 to HH 829 in both [SII] and $\mathrm{H} \alpha$. These objects are fainter in $\mathrm{H} \alpha$, with the exception of $\mathrm{HH} 827$ which is much stronger, while $\mathrm{HH} 826 \mathrm{~A}$ and $\mathrm{HH} 828$ are not seen at all in $\mathrm{H} \alpha$ emission. The errors in alignment of the central frame (HH 826, HH $220 \mathrm{NW}$ and $\mathrm{HH} 828$ ) in [SII] and $\mathrm{H} \alpha$ are quite high due to the low number of stars.

The PA of the blueshifted HH 220 jet is at $\sim 144^{\circ}$ with respect to CW Tau (Gomez de Castro 1993; Dougados et al. 2000) and we previously (MR04) measured the redshifted jet to be at $\sim 329^{\circ}$ from the images of Dougados et al. (2000). From Table 3 it can be seen that $\mathrm{HH} 826 \mathrm{~A}$, which is quite close to the source, is moving in a direction of $159^{\circ}$ i.e. it is well aligned with the blueshifted $\mathrm{HH} 220$ jet. $\mathrm{HH} 826 \mathrm{~B}$ is moving at $156^{\circ}$ in [SII] and $166^{\circ}$ in $\mathrm{H} \alpha$ and so is well aligned with both $\mathrm{HH} 826 \mathrm{~A}$ and the blueshifted jet. Similarly $\mathrm{HH} 826 \mathrm{C}$, moving at $321^{\circ}$ (in [SII]), is well aligned with the redshifted $\mathrm{HH} 220$ jet. This knot appears to be moving $\sim 65^{\circ}$ further east (at $26^{\circ}$ ) in $\mathrm{H} \alpha$.

There are two optically visible parts to the extended redshifted HH 220 jet. First, it extends to $9^{\prime \prime}$ from the source, there is then a gap before the second part of the jet is seen at $20^{\prime \prime}$ to $37^{\prime \prime}$ from the source, ending in HH 826C (MR04). The 9" long segment close to CW Tau was too faint to measure, however the longer HH $220 \mathrm{NW}$ (northwest) segment was measured in [SII] and has a direction of $287^{\circ}$. This direction is roughly aligned with $\mathrm{HH} 826 \mathrm{C}$ (at $321^{\circ}$ ) and with the PA of the redshifted "micro-jet" closer to the source $\left(329^{\circ}\right)$.

For the purpose of more accurate proper motions, we have divided HH 827 into Knot B (the brightest and largest part of HH 827) and Knot A (the closest bright knot to CW Tau) - see Fig. 1. HH 827 is much stronger in $\mathrm{H} \alpha$, and these proper motion studies show it to be moving at $184^{\circ}$ (this is an average for $\mathrm{HH} 827 \mathrm{~A}$ and $\mathrm{B}$ in $\mathrm{H} \alpha$ ). Although $\mathrm{HH} 827 \mathrm{~B}$ is much smaller and fainter in [SII], its direction of motion is still in good agreement with the $\mathrm{H} \alpha$ values at $198^{\circ}$. Knot $\mathrm{A}$ is too faint to measure in [SII]. Even taking errors into account, these directions rule out IRAS $04113+2758$ (see Fig. 1) as a possible source of HH 827 , as suggested in MR04. As we do not know if and where this outflow starts to bend beyond HH $826 \mathrm{~B}$ it is difficult to know if this object is part of the CW Tau outflow. It was suggested in MR04 that CW Tau could be driving this object if the outflow is precessing and these results are consistent with this scenario.

The velocity of $\mathrm{HH} 827 \mathrm{~B}$ is $199 \mathrm{~km} \mathrm{~s}^{-1}$ in [SII] and $151 \mathrm{~km} \mathrm{~s}^{-1}$ in $\mathrm{H} \alpha$. These values are just outside the associated errors. There appears to be a very large decrease in velocity from $347 \mathrm{~km} \mathrm{~s}^{-1}$ for Knot A to $151 \mathrm{~km} \mathrm{~s}^{-1}$ for Knot B.

The HH 828 knots to the north of CW Tau are quite faint and diffuse and their proper motions are not easily measured. The western and middle knots are moving at approximately $208^{\circ}$ and $144^{\circ}$ respectively while it was not possible to measure proper motions for the eastern knot. These directions rule out the possibility of them being driven by CW Tau, IRAS $04111+2804$ or IRAS 04108+2803 (see Fig. 1) as suggested in MR04. There are two candidate driving sources to the north of $\mathrm{HH} 828$ : FN Tau (a CTTS) and IRAS 04110+2820. HH 828 is $13^{\prime}$ at a PA of $184^{\circ}$ from FN Tau, and is 13.1 at a PA of $181^{\circ}$ from IRAS $04110+2820$. Both of these sources are just beyond the northern field of view of Fig. 1. The proper motion directions of the HH 828 knots suggest that they may by driven by either of these sources. However HH 828 is at a projected distance of $\sim 0.5 \mathrm{pc}\left(\sim 13^{\prime}\right)$ and at that distance from these possible sources we would expect that only the largest, most chaotic shocks survive.

Turning to $\mathrm{HH} 829$, knot $\mathrm{C}$ is too faint to measure in both [SII] and $\mathrm{H} \alpha$ and knots $\mathrm{A}$ and $\mathrm{B}$ were measured in [SII] only. Knot $\mathrm{A}$ is moving in a direction of $108^{\circ}$ and $\mathrm{B}$ is at $163^{\circ}$, ruling out CW Tau as their driving source. It can be seen from Fig. 1 that HH $829 \mathrm{~B}$ has a similar direction of motion to that of the HH 828 knots, suggesting a common driving source. HH $829 \mathrm{~B}$ is 1 '.8 from FN Tau and 1' from IRAS $04110+2820$. It is possible that HH $829 \mathrm{~B}$ is part of an outflow driven by FN Tau or IRAS $04110+2820$ (as mentioned earlier in respect to HH 828). There is no evidence of jets emanating from either source.

It can be seen from the results in Table 3 that the velocity measurements can be quite different for individual knots in $\mathrm{H} \alpha$ and [SII]. However the difference in [SII] and $\mathrm{H} \alpha$ proper motion vectors for many of the objects are within errors. For example, $\mathrm{HH} 826 \mathrm{~B}$ has a velocity and position angle difference of $75 \mathrm{~km} \mathrm{~s}^{-1}$ and $10^{\circ}$. The errors for this frame are quite large as mentioned earlier (due to the small number of stars) i.e. $\pm 55 \mathrm{~km} \mathrm{~s}^{-1}$ for both the [SII] and $\mathrm{H} \alpha$ measurement while the sum of the errors in position angle is $26^{\circ}$. For $\mathrm{HH} 827 \mathrm{~B}$, the difference in velocity measurements is just outside the associated errors while that of the position angle is within the errors. HH $826 \mathrm{~A}$, however, has substantial differences between its [SII] and $\mathrm{H} \alpha$ measurement. This point is further discussed in Sect. 5.3. For the rest of the $\mathrm{HH}$ objects listed in Table 3 there are only values for either [SII] or $\mathrm{H} \alpha$. 


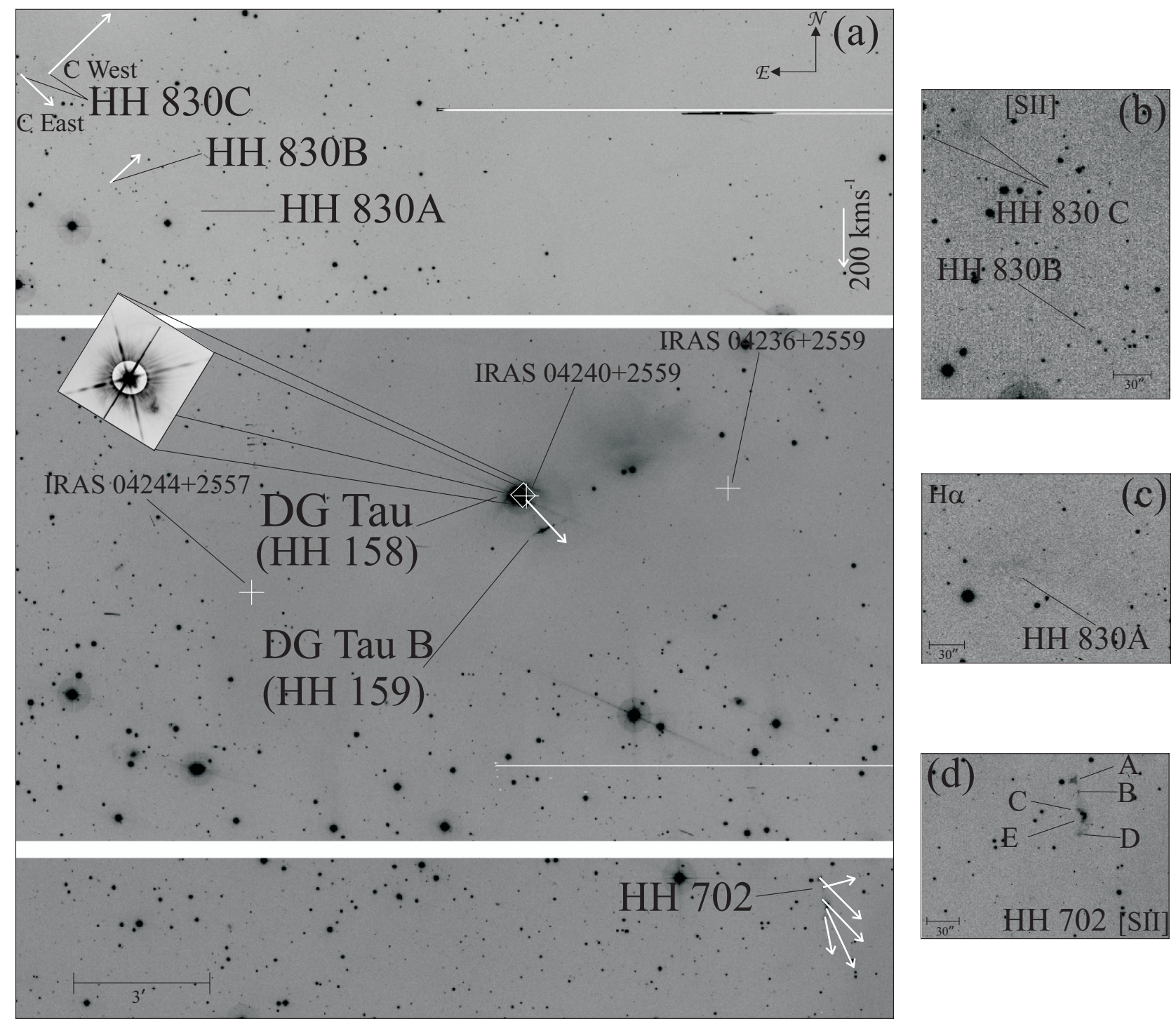

Fig. 2. a) Mosaic image showing proper motions of HH 702 and HH 830 in the vicinity of DG Tau in [SII]. The direction of motion of each $\mathrm{HH}$ object is represented by white arrows. The relative length of these arrows denotes the relative velocity of the object. Our study shows that HH 702 is likely to be driven by DG Tau, but that HH 830 is not. HH $830 \mathrm{C}$ (West) and B appear to be driven by a source to the east however we are unable to suggest any. As $\mathrm{HH} 830 \mathrm{C}$ is on the edge of the mosaic, its proper motion is possibly less precise. A HST image of HH 158 is included here as an inset. b) $\mathrm{HH} 830 \mathrm{~B}$ and $\mathrm{C}$ in [SII]. The error in velocity for these objects are high $\left( \pm 60 \mathrm{~km} \mathrm{~s}^{-1}\right.$ in [SII] and $\pm 40 \mathrm{~km} \mathrm{~s}^{-1}$ in $\mathrm{H} \alpha$, see Sect. 3) as they are very faint. c) $\mathrm{HH} 830 \mathrm{~A}$ is stronger in $\mathrm{H} \alpha$ than in [SII], however it is still too faint to do proper motion studies on. d) Knots A-E of HH 702 (see MR04).

While this proper motion study confirms that $\mathrm{HH} 826$ and HH 827 are likely driven by CW Tau, it shows that HH 828 and HH 829 are not. This reduces the known projected length of the outflow from $0.98 \mathrm{pc}\left(24^{\prime}\right)$, as suggested in MR04, to $0.32 \mathrm{pc}$ (7:7), which is still much greater than the $\sim 0.008 \mathrm{pc}\left(\sim 12^{\prime \prime}\right)$ long "micro-jet" that was previously seen.

\section{2. $D G T a u$}

HH 158 is a "micro-jet" close to DG Tau that was originally discovered by Mundt \& Fried (1983) and extends for $~ 16^{\prime \prime}$ (Eislöffel \& Mundt 1998) at a PA of $223^{\circ}$ (Bacciotti et al. 2002). In MR04 we suggested that the DG Tau outflow is much longer and may also consist of two other HH complexes which are approximately aligned with $\mathrm{HH} 158$ - HH 702 which was also independently discovered by Sun et al. (2003) and HH 830 (see Fig. 2). If this were the case the DG Tau outflow would actually extend for at least $27^{\prime}(\sim 1.1 \mathrm{pc})$. Here we present proper motion studies confirming that HH 702 is indeed driven by DG Tau, however our studies of $\mathrm{HH} 830$ suggest it is not.

Table 4 shows the velocity and direction of motion of knots A-E in HH 702. In [SII] emission knots A, C, D and E are moving at between $\sim 191^{\circ}$ and $\sim 225^{\circ}$. These knots are well aligned with $\mathrm{HH} 158$ which has as a PA of $223^{\circ}$ with respect to DG Tau (see Fig. 2). Knot B appears to be moving at $285^{\circ}$ and so is not aligned with the other knots in HH 702. Knots A, $\mathrm{C}$ and D have comparable velocities between $\sim 203 \mathrm{~km} \mathrm{~s}^{-1}$ and $\sim 219 \mathrm{~km} \mathrm{~s}^{-1}$ while knots $\mathrm{B}$ and $\mathrm{E}$ have velocities of only approximately half of this, at $97 \mathrm{~km} \mathrm{~s}^{-1}$ and $125 \mathrm{~km} \mathrm{~s}^{-1}$ respectively. 
Table 4. Tangential velocity and direction of motion of HH 158, HH 702 and HH 830 in the vicinity of DG Tau assuming a distance of 140 pc (Elias 1978; Wichmann et al. 1998). The associated errors for HH 830 are $\pm 60 \mathrm{~km} \mathrm{~s}^{-1}$ in [SII] and $\pm 40 \mathrm{~km} \mathrm{~s}^{-1}$ in $\mathrm{H} \alpha$ images. For HH 702 the errors are $\pm(10-28) \mathrm{km} \mathrm{s}^{-1}$ (see Sect. 3). All errors are $1 \sigma$.

\begin{tabular}{|c|c|c|c|c|c|c|}
\hline \multirow[b]{2}{*}{ HH object } & \multirow[b]{2}{*}{ Source } & \multirow[b]{2}{*}{$\begin{array}{l}\text { Ang. } \\
\text { Sep. }\end{array}$} & \multicolumn{2}{|c|}{ [SII] } & \multicolumn{2}{|r|}{$\mathrm{H} \alpha$} \\
\hline & & & $\begin{array}{l}\text { Velocity } \\
/ \mathrm{km} \mathrm{s}^{-1}\end{array}$ & $\begin{array}{c}\text { Direction } \\
/^{\circ}\end{array}$ & $\begin{array}{l}\text { Velocity } \\
/ \mathrm{km} \mathrm{s}^{-1}\end{array}$ & $\begin{array}{c}\text { Direction } \\
/^{\circ}\end{array}$ \\
\hline $\mathrm{HH} 158 \mathrm{C}^{a}$ & DG Tau & $12^{\prime \prime}$ & 197 & 223 & & \\
\hline HH 702 A & DG Tau & $10{ }^{\prime} 6$ & 203 & $225 \pm(3-8)$ & 129 & $194 \pm(5-12)$ \\
\hline HH 702 B & DG Tau & $10^{\prime} 9$ & 97 & $285 \pm(3-8)$ & & \\
\hline HH $702 \mathrm{C}$ & DG Tau & 11 '.1 & 204 & $224 \pm(6-17)$ & 149 & $128 \pm(4-11)$ \\
\hline HH 702 D & DG Tau & $11: 4$ & 219 & $205 \pm(3-8)$ & 190 & $269 \pm(3-9)$ \\
\hline HH 702 E & DG Tau & 11.4 & 125 & $191 \pm(3-7)$ & 186 & $117 \pm(3-9)$ \\
\hline HH 830 C West & & & 298 & $314 \pm 28$ & & \\
\hline HH 830 C East & & & 161 & $226 \pm 12$ & 348 & $314 \pm 7$ \\
\hline HH 830 B & & & 142 & $313 \pm 22$ & 327 & $233 \pm 7$ \\
\hline
\end{tabular}

${ }^{a}$ Proper motions could not be obtained by us for HH 158 (see text). Angular separation and velocity values are taken from Eislöffel \& Mundt (1998) and the direction of motion of HH 158 is taken from Bacciotti et al. (2002).

It can be clearly seen from Fig. 2 that knots A to E (excluding B) are moving in a direction that would be expected if DG Tau is the driving source. Hence, we can rule out the possibility that knot B has a different source.

The direction of motion found in $\mathrm{H} \alpha$ for the HH 702 knots range from $117^{\circ}$ for knot $\mathrm{E}$ to $269^{\circ}$ for knot $\mathrm{D}$. Knot $\mathrm{A}$ is moving within $\sim 30^{\circ}$ of the direction found in [SII], which is outside the associated errors for the two measurements, while knots C, D and $\mathrm{E}$ are significantly different. Knots $\mathrm{A}$ and $\mathrm{C}$ have comparable velocities of $\sim 129 \mathrm{~km} \mathrm{~s}^{-1}$ and $149 \mathrm{~km} \mathrm{~s}^{-1}$ in $\mathrm{H} \alpha$ while knots D and $\mathrm{E}$ are also comparable at $\sim 186 \mathrm{~km} \mathrm{~s}^{-1}$ and $190 \mathrm{~km} \mathrm{~s}^{-1}$. Only knot D has comparable velocity in both $\mathrm{H} \alpha$ and [SII] of $190 \mathrm{~km} \mathrm{~s}^{-1}$ and $215 \mathrm{~km} \mathrm{~s}^{-1}$. Knot B has a much slower velocity of $45 \mathrm{~km} \mathrm{~s}^{-1}$ in $\mathrm{H} \alpha$.

For the HH 702 knots A, C, D and E we have obtained proper motions in both $\mathrm{H} \alpha$ and [SII] emission lines. HH 702 B is too faint to get proper motions in $\mathrm{H} \alpha$. Calculating the average velocities and direction of motions for these four knots yields comparable values of $188 \mathrm{~km} \mathrm{~s}^{-1}$ at $211^{\circ}$ in [SII] and $164 \mathrm{~km} \mathrm{~s}^{-1}$ at $177^{\circ}$ in $\mathrm{H} \alpha$. The direction of motion for $\mathrm{HH} 702$ knots A-E is in very good agreement with the PA of the $\mathrm{HH} 158$ jet at $223^{\circ}$.

HH 830 to the northeast of DG Tau consists of knots A, B and $\mathrm{C}$, with knot $\mathrm{C}$ containing two separate emission regions (C West and C East). Table 4 shows the results of the proper motion study of $\mathrm{HH} 830 \mathrm{~B}$ and $\mathrm{C}$ only as knot $\mathrm{A}$ is too faint to measure. Figure 2 shows that knots $\mathrm{B}$ and $\mathrm{C}$ West are moving in parallel. Knot $\mathrm{C}$ East is moving orthogonally to these however this knot is at the eastern edge of the field of view of the WFC CCD mosaic which makes it more difficult to determine accurate proper motions. Also, as mentioned in Sect. 3, the errors in the proper motions of $\mathrm{HH} 830$ in both [SII] and $\mathrm{H} \alpha$ are quite high. These direction of motions prove that these knots are not driven by DG Tau. Therefore the projected length of the DG Tau outflow must be revised downwards to $0.5 \mathrm{pc}$ (12.33).

We were unable to obtain proper motions for HH 158 from our images due to the significant amount of nebulosity surrounding this object. Eislöffel \& Mundt (1998) determined the velocity of Knot C of HH 158 as $197 \mathrm{~km} \mathrm{~s}^{-1}$. The results from our proper motion study show that the velocity of $\mathrm{HH} 702$ is comparable to that of HH 158 (both in [SII]), despite the 7:7 (0.3 pc projected) gap between these two objects. This point is discussed further in Sect. 5.4.

\subsection{The DO Tau and HV Tau $C$ region}

The $2^{\prime \prime}-4$ " long bipolar HH 230 "micro-jet" at a PA of $\sim 70^{\circ}$ with respect to DO Tau was first observed by Hirth et al. (1994). Two newly discovered HH objects, HH 831 and HH 832 (see MR04), to the east of DO Tau appear to be well aligned with HH 230 (see Fig. 3), suggesting that they are part of this outflow. However this proper motion study shows the HH 831 knots (A and $\mathrm{B}$ ) to be moving in a completely different direction than that expected if driven by DO Tau.

HH 831 A knots A1, A2, A3 and A5 are moving in similar directions between $284^{\circ}$ and $331^{\circ}$ (Table 5) in [SII]. Knot A4 is too faint to measure in [SII]. B1 and B2 (only measured in [SII]) however are moving in a different direction at $158^{\circ}$ and $134^{\circ}$ respectively. Only knots $\mathrm{A} 4$ and $\mathrm{A} 5$ could be measured in $\mathrm{H} \alpha$ however the directions of knot $\mathrm{A} 4$ at $32^{\circ}$ is not aligned with any of the directions seen in [SII] and knot A5 is moving at $182^{\circ}$ in $\mathrm{H} \alpha$, which is not aligned with its [SII] results. As the measurable motions in $\mathrm{H} \alpha$ are very different from [SII] and the rest of HH $831 \mathrm{~A}$ and $\mathrm{HH} 831 \mathrm{~B}$ are too faint to be detected in $\mathrm{H} \alpha$ it would appear that the $\mathrm{H} \alpha$ results are not reliable.

The velocities of HH 831 A1, A2 and A3 (in [SII]) are comparable and range from $166 \mathrm{~km} \mathrm{~s}^{-1}$ to $196 \mathrm{~km} \mathrm{~s}^{-1}$ with knot A5 slower at $112 \mathrm{~km} \mathrm{~s}^{-1}$. Knots B1 and B2 have comparable velocities of $71 \mathrm{~km} \mathrm{~s}^{-1}$ and $79 \mathrm{~km} \mathrm{~s}^{-1}$ respectively.

From these results it is obvious that HH 831 is not driven by DO Tau, despite the fact that these knots appear to be very well aligned with the known PA of HH 230 (Fig. 3). These results show a number of different directions of motion within HH 831 knots A1, A2, A3 and A5 (in [SII]) are moving west/northwest, while knots B1 and B2 are moving to the southeast. Knot A4 (in $\mathrm{H} \alpha$ ) appears to be separate to all of these and is moving to the northeast. Possible driving sources for HH $831 \mathrm{~A}$ based on these proper motion results will be discussed at the end of this section. It is possible that either of the two nearby LDN 1527 radio sources (Anglada et al. 1992) could be driving HH 831 B1 and B2 (see Fig. 3). HH 832, which is seen in [SII] only, is too faint to measure its proper motion.

The proper motions of $\mathrm{HH} 831 \mathrm{~A}$ (between $284^{\circ}$ and $331^{\circ}$ ) may suggest a common source to its east. There are six known candidates. The distance and PA of $\mathrm{HH} 831$ A relative to each of these is given below followed by a discussion on the most likely source:

1. ITG 14, a YSO (Itoh et al. 1999) (6' at a PA of $\left.275^{\circ}\right)$.

2. IRAS $04365+2605\left(6.5\right.$ at a PA of $\left.291^{\circ}\right)$. 

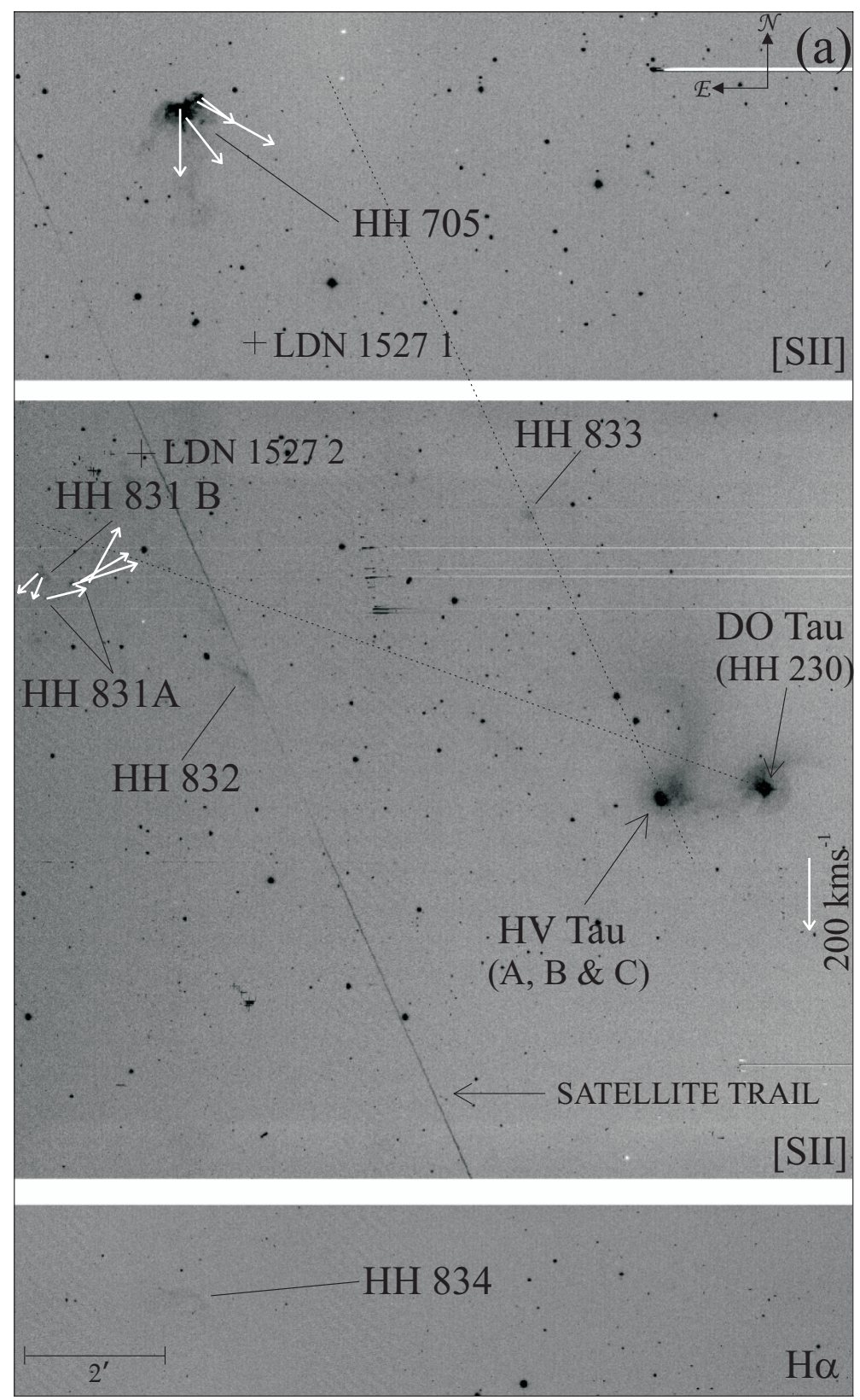
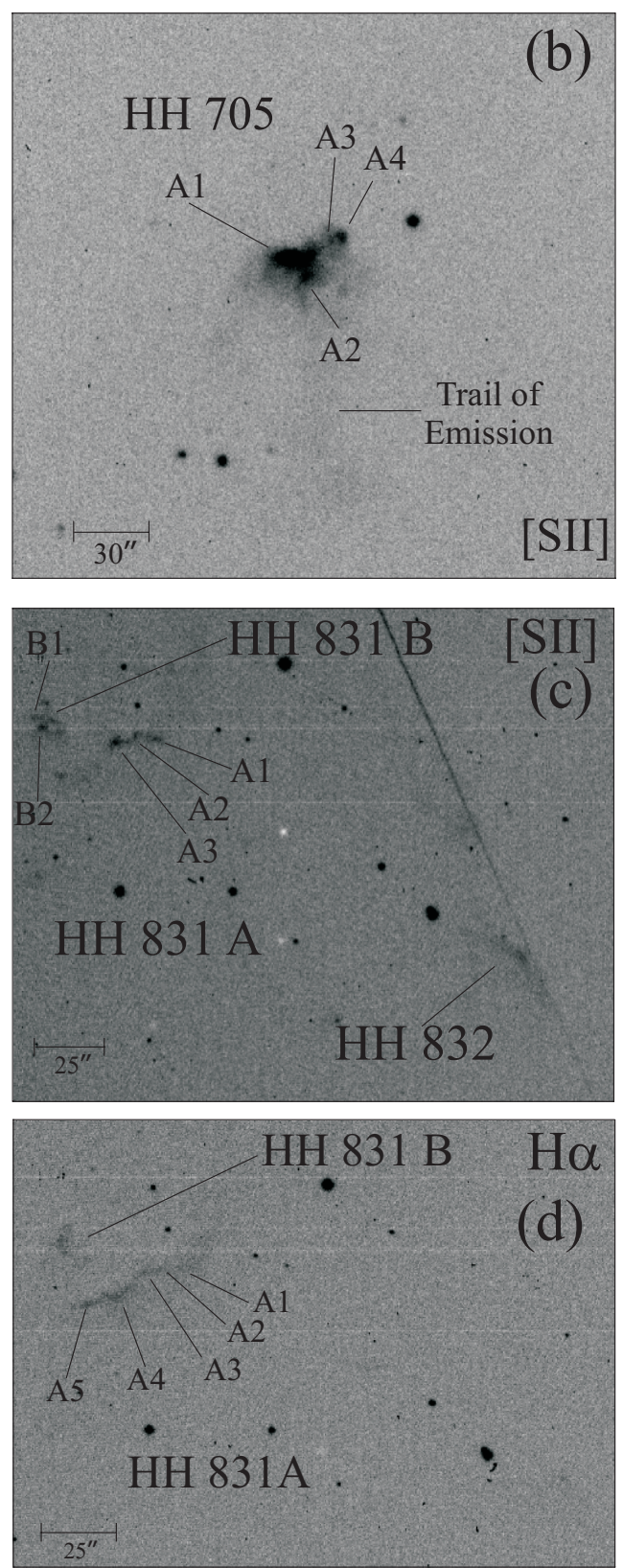

Fig. 3. a) Mosaic image showing proper motion vectors for the HH objects in the vicinity of DO Tau and HV Tau C. The direction of motion of each $\mathrm{HH}$ object is represented by white arrows. The relative length of these arrows denotes the relative velocity of the object. These vectors show, surprisingly, that neither of these sources are driving HH 831 and $\mathrm{HH} 705$ as was suggested in MR04. No proper motions could be detected for the fainter objects $\mathrm{HH} 832, \mathrm{HH} 833$ and $\mathrm{HH} 834$, however it is still possible that $\mathrm{HH} 832$ may be driven by DO Tau based on its rough alignment with DO Tau's jet. The dashed line from DO Tau is at $70^{\circ}$ which is the PA of HH 230 while the dashed line from HV Tau C is at $25^{\circ}$, marking the PA of its "micro-jet". Candidate driving sources for HH 831, HH 833 and HH 705 are discussed in the text. Images b)-d) show the individual knots in HH 705 and HH 831 for which the proper motion vectors were calculated.

3. IRAS $04371+2559\left(16^{\prime} .8\right.$ at a PA of $\left.299^{\circ}\right)$.

4. IRAS $04370+2559\left(15: 7\right.$ at a PA of $\left.302^{\circ}\right)$.

5. IRAS $04368+2557\left(13.8\right.$ at a PA of $\left.312^{\circ}\right)$.

6. IRAS $04366+2556\left(13.5\right.$ at a PA of $\left.325^{\circ}\right)$.

The most likely source is IRAS $04368+2557$, which has previously been associated with HH 192 A, B and C (Eiroa et al. 1994; Gomez et al. 1997). From the images of Gomez et al. (1997) $\mathrm{HH} 192 \mathrm{~A}$ and B are aligned with the blue lobe of a molecular outflow and extend for $\sim 2^{\prime}$ from the IRAS source at a PA of $\sim 80^{\circ}$. HH $192 \mathrm{C}$ is aligned with the redshifted lobe of the molecular outflow and is $\sim 4.2$ from IRAS $04368+2557$ at a PA of $277^{\circ}$. If the outflow is precessing HH $831 \mathrm{~A}$ could be part of it.

A 1".5 long bipolar "micro-jet" was recently discovered from HV Tau C by Stapelfeldt et al. (2003), which we estimate to be at a PA of $25^{\circ}$ (northern jet) from their images (MR04). HH 833, to the northeast of $\mathrm{HV}$ Tau $\mathrm{C}$, is also at a PA of $25^{\circ}$ suggesting that it may also be driven by this source (see Fig. 3). Further out in this direction is HH 705 (independently discovered by Sun et al. 2003) which may also be part of this outflow. Due to morphological alignment we suggested in MR04 that LDN 15271 and LDN 15272 which lie to the south of HH 705 are other possible 
Table 5. Tangential velocity and direction of motion of HH 831 and HH 705 assuming a distance of 140 pc (Elias 1978; Wichmann et al. 1998) to the Taurus Auriga cloud. The associated errors for HH 831 and HH 705 are $\pm(10-28) \mathrm{km} \mathrm{s}^{-1}$ (see Sect. 3). All errors are $1 \sigma$.

\begin{tabular}{lcccccc}
\hline \hline HH Object & Source & $\begin{array}{c}\text { Ang. } \\
\text { Sep. }\end{array}$ & $\begin{array}{c}\text { Velocity } \\
/ \mathrm{km} \mathrm{s}^{-1}\end{array}$ & $\begin{array}{c}\text { Direction } \\
{ }^{\circ}\end{array}$ & $\begin{array}{c}\mathrm{H} \alpha \\
\text { Velocity } \\
/ \mathrm{km} \mathrm{s}^{-1}\end{array}$ & $\begin{array}{c}\text { Direction } \\
/{ }^{\circ}\end{array}$ \\
\hline HH 831 A1 & $a$ & & 184 & $331 \pm(3-9)$ & & \\
HH 831 A2 & $a$ & & 166 & $300 \pm(4-10)$ & & \\
HH 831 A3 & $a$ & & 196 & $288 \pm(3-8)$ & & $32 \pm(4-11)$ \\
HH 831 A4 & & & & & 141 & $182 \pm(3-7)$ \\
HH 831 A5 & $a$ & & 112 & $284 \pm(5-14)$ & 221 & \\
HH 831 B1 & & 71 & $158 \pm(8-22)$ & & \\
HH 831 B2 & & 79 & $134 \pm(8-20)$ & & \\
HH 705 A1 & & 187 & $180 \pm(3-9)$ & 330 & $186 \pm(2-5)$ \\
HH 705 A2 & & 147 & $218 \pm(4-11)$ & 231 & $186 \pm(3-7)$ \\
HH 705 A3 & & 244 & $241 \pm(3-7)$ & 194 & $131 \pm(3-8)$ \\
HH 705 A4 & & 99 & $232 \pm(6-16)$ & 211 & $227 \pm(3-8)$ \\
\hline
\end{tabular}

${ }^{a}$ The driving source is currently unknown however three IRAS sources are suggested in the text as possible sources for HH 831 A1-A3 and A5.

driving sources of HH 705 (MR04; Sun et al. 2003) but we suggested that it was unlikely that LDN 15271 could generate such a large object so nearby.

Proper motion studies of the HH 833 and HH 705 objects did not reveal anything conclusive about their sources. HH 833 was too faint to measure, so we still suggest that it is driven by HV Tau $\mathrm{C}$ due to its exact alignment with the HV Tau $\mathrm{C}$ jet (however see below for an alternative driving source). Figure 3 shows the direction of motion of the HH 705 knots which are detailed in Table 5. All knots in HH 705 (A1-A4) are moving to the south/southwest in [SII]. The $\mathrm{H} \alpha$ results show a similar direction of motion however A3 is moving in a more southwesterly direction $\left(\right.$ at $131^{\circ}$ ). The southward motions of HH 705 conclusively rule out HV Tau C, LDN 15271 or LDN 15272 as its source (see MR04 and Fig. 3). There is an IRAS source, IRAS 04358+2618, $\sim 5^{\prime}(1.5 \mathrm{pc})$ to the north of HH 705 which remains the only known possible driving source for this object. HH 705 is at a PA of $213^{\circ}$ with respect to this source which is well aligned with the proper motion of the $\mathrm{HH} 705$ knots which are between $180^{\circ}$ and $241^{\circ}$. HH 833 is at a PA of $221^{\circ}$ with respect to HH 705 showing that IRAS $04358+2618$ could be driving a $\sim 12$ ! 7 long outflow consisting of $\mathrm{HH} 705$ and $\mathrm{HH} 833$.

The HH 705 knots A1-A4 have been measured in both [SII] and $\mathrm{H} \alpha$. While the individual knots show different velocities and direction of motion, if we get the average for both of these emission lines the proper motions are presumably more reliable: $169 \mathrm{~km} \mathrm{~s}^{-1}$ at $218^{\circ}$ in [SII] and $242 \mathrm{~km} \mathrm{~s}^{-1}$ at $183^{\circ}$ in $\mathrm{H} \alpha$.

Our proper motion studies have shown that HH705 and HH 831 are not driven by DO Tau or HV Tau C. In MR04 we suggested that these CTTSs were driving parsec-scale outflows however our proper motion study shows that this is not the case.

\subsection{RW Aur}

The HH 229 jet from RW Aur was first noted by Hamann (1994) and Hirth et al. (1994). The outflow is at a PA of $130^{\circ}$ (Dougados et al. 2000; Mundt \& Eislöffel 1998; Hirth et al. 1997) with respect to RW Aur and is at least $106^{\prime \prime}$ in length while the redshifted outflow is at least 50" long (Mundt \& Eislöffel 1998). HH 835 is 5.37 from RW Aur at a PA of $310^{\circ}$ which is exactly aligned with the redshifted HH 229 jet (see Fig. 4). HH 835 is much stronger in $\mathrm{H} \alpha$ than [SII] and proper motions were only determined for $\mathrm{H} \alpha$. In MR04 we suggested that it is a bow shock with only the northern wing visible here. If $\mathrm{HH} 835$ is part of this

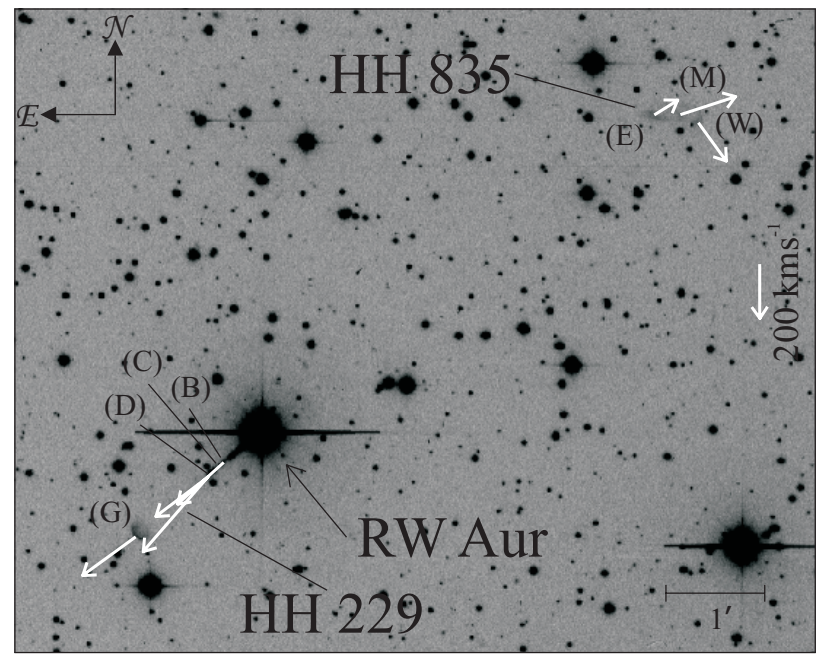

Fig. 4. Proper motion vectors for HH835 and HH229 in the RW Aur outflow in $\mathrm{H} \alpha$. The direction of motion of each $\mathrm{HH}$ object is represented by white arrows. The relative length of these arrows denotes the relative velocity of the object. The direction of motion of the eastern and middle knots of HH 835 show RW Aur to be driving this object. Proper motions were also measured for four knots in the blueshifted HH 229 outflow.

outflow then the total projected length of this outflow from the blueshifted HH 229 jet to HH 335 is 0.29 pc $\left(7^{\prime}\right)$.

HH 835 appears to consist of a number of discrete objects which we refer to here as the west (W), middle (M) and east (E) knots in Fig. 4 and Table 6. Of these, the eastern knot appears to be the brightest. Proper motion studies show this knot to be moving in a direction of $302^{\circ}$ (Table 6) which is well aligned with the PA of the redshifted HH 229 jet. The motion of the middle knot is also closely aligned at $288^{\circ}$. The western knot however is at $216^{\circ}$ and so appears to be moving in a different direction. Overall, these proper motion vectors confirm the suggestion that this object is part of the RW Aur outflow. In MR04 we suggested that HH 835 may be one side of a bow shock, with the other side being optically obscured. In this case, knot W would be at the head of the bow shock with knots $\mathrm{M}$ and $\mathrm{E}$ further back along one side of the shock. The velocities of knots $\mathrm{W}$ and $\mathrm{M}$ are $162 \mathrm{~km} \mathrm{~s}^{-1}$ and $208 \mathrm{~km} \mathrm{~s}^{-1}$ while knot $\mathrm{E}$ is has a much lower velocity of $92 \mathrm{~km} \mathrm{~s}^{-1}$. These values are consistent with a slowing 
Table 6. Tangential velocity and direction of motion of HH 835 and HH 229 in the RW Aur outflow assuming a distance of $140 \mathrm{pc}$ to the source (Elias 1978; Wichmann et al. 1998). HH 835 is very weak in [SII] so proper motion studies could only be done with the $\mathrm{H} \alpha$ images. The nomenclature for the knots in HH 229 are taken from Eislöffel \& Mundt (1998). The associated errors for HH 229 and HH 835 are $\pm(10-28) \mathrm{km} \mathrm{s}^{-1}$ (see Sect. 3). All errors are $1 \sigma$.

\begin{tabular}{|c|c|c|c|c|}
\hline \multirow[b]{2}{*}{ HH Object } & \multirow[b]{2}{*}{ Source $^{a}$} & \multirow[b]{2}{*}{$\begin{array}{l}\text { Ang. } \\
\text { Sep. }\end{array}$} & \multicolumn{2}{|r|}{$\mathrm{H} \alpha$} \\
\hline & & & $\begin{array}{l}\text { Velocity } \\
/ \mathrm{km} \mathrm{s}^{-1}\end{array}$ & $\begin{array}{c}\text { Direction } \\
/^{\circ}\end{array}$ \\
\hline HH $835 \mathrm{E}$ & RW Aur $(R)$ & 4.5 & 92 & $302 \pm(6-17)$ \\
\hline $\mathrm{HH} 835 \mathrm{M}$ & RW Aur $(R)$ & $4^{\prime} .6$ & 208 & $288 \pm(3-8)$ \\
\hline HH 835 W & RW Aur $(R)$ & 4.7 & 162 & $216 \pm(4-10)$ \\
\hline HH $229 \mathrm{~B}$ & RW Aur $(B)$ & 0.4 & 223 & $132 \pm(3-7)$ \\
\hline HН $229 \mathrm{C}$ & RW Aur $(B)$ & 0.5 & 307 & $139 \pm(2-5)$ \\
\hline HH $229 \mathrm{D}$ & RW Aur $(B)$ & 0.6 & 238 & $127 \pm(3-7)$ \\
\hline HH $229 \mathrm{G}$ & RW Aur $(B)$ & 1.6 & 235 & $126 \pm(3-7)$ \\
\hline
\end{tabular}

${ }^{a} B$ and $R$ denotes whether the object is aligned with the blue or redshifted jet.

down in velocity of knots along the edge of the bow shock away from the head.

Proper motions were also obtained for some of the brighter knots in the HH 229 blueshifted outflow. Knots B, C, D and G (using the nomenclature of Eislöffel \& Mundt 1998) were strong enough in $\mathrm{H} \alpha$ to determine their tangential velocity and direction - see Table 6. Knots B, D and G can be seen from Fig. 4 to be moving along the optically visible jet direction at $132^{\circ}, 127^{\circ}$ and $126^{\circ}$ respectively, while knot $\mathrm{C}$ is slightly off this direction at $139^{\circ}$. The velocities of knots B, D and G are comparable, ranging from $223 \mathrm{~km} \mathrm{~s}^{-1}$ to $238 \mathrm{~km} \mathrm{~s}^{-1}$ while knot $\mathrm{C}$ is faster at $307 \mathrm{~km} \mathrm{~s}^{-1}$. The redshifted counterflow was too faint to measure. The known angular extent of the RW Aur outflow remains at $7^{\prime}$ (0.29 pc).

\section{Discussion}

\subsection{Determining outflow sources}

The proper motion studies undertaken here have confirmed the sources suggested in MR04 for a number of $\mathrm{HH}$ objects e.g. HH 826 and HH 827 (CW Tau); HH 702 (DG Tau) and HH 835 (RW Aur). However they have also refuted the suggested sources for others - HH 828, HH 829, HH 830, HH 831 and HH 705. This was surprising in some cases as the objects were well aligned with known outflows - this is most noticeable in the case of DO Tau where HH 831 appeared to be an extension of the HH 230 outflow based on their close alignment. In these cases, we have tried to suggest candidate sources based on their newly discovered direction of motion.

These proper motion studies highlight the need for circumspection in using the apparent alignment of $\mathrm{HH}$ objects to derive their driving source. Nevertheless, in the absence of proper motion studies apparent outflow alignment is still the best means of finding potential driving sources. Proper motion studies can then conclusively confirm or refute these sources at a later date. As the CTTSs examined in this paper are relatively close by at $140 \mathrm{pc}$, a time difference between observations of just a few years is sufficient to accurately measure their motion.

\subsection{Parsec-scale outflows from CTTSs}

In MR04 we suggested that five CTTS sources (CW Tau, DG Tau, DO Tau, HV Tau C and RW Aur) drive large-scale outflows, of the order of $0.5 \mathrm{pc}-1 \mathrm{pc}$, based on distant $\mathrm{HH}$ objects that appeared morphologically and/or by position to be part of the outflow. However in light of the present study we now know that only three of these stars definitely do: CW Tau drives a $7.7(0.32 \mathrm{pc})$ outflow, DG Tau drives a 12 !.3 (0.5 pc) outflow while the known angular extent of the RW Aur outflow is still 7' $(0.29 \mathrm{pc})$. While the length of these outflows is at the lower limit of those suggested in MR04, they are still much larger than the "micro-jets" they were previously known to drive. HV Tau may still be associated with $\mathrm{HH} 833$ which was too faint to measure its proper motion.

The CW Tau and DG Tau outflows appear very much onesided in that the blue side is extended while the red side appears very, very short. It is not uncommon for the red side of an outflow to be hidden from view as it recedes from us into the cloud, so it is very likely that the CW Tau and DG Tau outflows are more extended in the red direction than our images show.

\section{3. [SII] versus $H \alpha$ velocities}

Proper motions were determined in both $[\mathrm{SII}]$ and $\mathrm{H} \alpha$ for the majority of $\mathrm{HH}$ objects here, the main exception being HH 835 and HH 229 where the individual knots were too weak in [SII] images to measure. There are few proper motion studies which calculate proper motions for both [SII] and $\mathrm{H} \alpha$ simultaneously and of these some find velocity measurements which are very similar e.g. HH 34 (Reipurth et al. 2002) while others find large variations for some knots e.g. HH 157 driven by FS Tau B (Eislöffel $\&$ Mundt 1998). Our results show varying degrees of agreement between $\mathrm{H} \alpha$ and [SII]; some knots have comparable velocities/directions of motion in these two emission lines, while others are quite different. However some of these differences can be understood in terms of errors in their measurements. Also, Hartigan et al. (2001) note that brightness changes between two different epochs will affect the $\mathrm{H} \alpha$ image more than the $[\mathrm{SII}]$ as $\mathrm{H} \alpha$ responds immediately to changes in the preshock density while [SII] averages this variability over the $\sim 30$ year cooling time. The different timescales over which cooling acts may explain some of the discrepancies in the results presented here.

\subsection{Velocity of distant HH objects}

In the introduction, we mentioned that the tangential velocity of objects at relatively large distances from their sources is currently not well known. In MR04 the dynamical timescales of these outflows were calculated using an assumed tangential velocity of $50 \mathrm{~km} \mathrm{~s}^{-1}$, comparable to the lowest velocity necessary to collisionally excite the shocks observed. This assumed velocity yielded dynamical ages of $0.6 \times 10^{4}-2.1 \times 10^{4}$ years. However from examining Tables 3, 4 and 6 we see that the velocity of the more distant objects is much higher than the $50 \mathrm{~km} \mathrm{~s}^{-1}$ lower limit. HH 827 (driven by CW Tau) has a value of $249 \mathrm{~km} \mathrm{~s}^{-1}$ (average of Knots A and B) in $\mathrm{H} \alpha$ and $199 \mathrm{~km} \mathrm{~s}^{-1}$ (Knot B) in [SII]. Knots A, C, D and E of HH 702 (driven by DG Tau) have an average velocity of $188 \mathrm{~km} \mathrm{~s}^{-1}$ in [SII] and of $164 \mathrm{~km} \mathrm{~s}^{-1}$ in $\mathrm{H} \alpha$. Finally HH 835 (driven by RW Aur) has an average velocity of $154 \mathrm{~km} \mathrm{~s}^{-1}$. Examination of these values would suggest that $200 \mathrm{~km} \mathrm{~s}^{-1}$ is more typical for relatively distant $\mathrm{HH}$ objects driven by CTTSs. 
This study has also shown that the lengths of these outflows are shorter than previously suggested, as some of the $\mathrm{HH}$ objects that appeared to be associated with the CTTSs are shown here to be unrelated. Re-calculating dynamical timescales to take account of these results reduces them to $1.4 \times 10^{3} \mathrm{yr}$ for CW Tau, $2.3 \times 10^{3} \mathrm{yr}$ for DG Tau and $0.9 \times 10^{3} \mathrm{yr}$ for RW Aur. However we stress that these are much lower than the actual outflow timescales (McGroarty et al. 2004; MR04).

\subsection{Velocity of "micro-jets"}

Out of the five "micro-jets" examined here HH 220, HH 158 and HH 229 are optically visible in our images and their proper motions could be measured. We find velocities of $323 \mathrm{~km} \mathrm{~s}^{-1}$ for HH 220 (NW) and $251 \mathrm{~km} \mathrm{~s}^{-1}$ for HH 229 (average of knots B, C, D and G) while HH 158 (A, B and C) have an average velocity of $241 \mathrm{~km} \mathrm{~s}^{-1}$ (Eislöffel \& Mundt 1998). These velocities are comparable to those found for jets from Class I sources, which are typically 200-400 $\mathrm{km} \mathrm{s}^{-1}$ (Mundt et al. 1987). This suggests that the velocity of the jets remains high as their sources evolve, despite the fact that the rate of accretion and ejection is about 10-100 times smaller for Class II sources than Class I YSOs (Hartigan et al. 1995).

Comparing our tangential velocity measurements for the distant objects to those of the "micro-jets" shows that the velocities are comparable despite the large difference in distance from their source. We cannot, of course, conclude that there is little or no deceleration in the outflow over parsec-scale distances as the "events" that give rise to the more distant $\mathrm{HH}$ objects could have originated from more violent (e.g. FUor) outbursts that those closer to the source.

There are two scenarios to be examined:

1. the ejection velocity at the source was much higher $\sim 10^{3}$ years ago when the more distant objects were ejected and has decreased over the intervening years to its current velocity of $200-300 \mathrm{~km} \mathrm{~s}^{-1}$. Meanwhile the velocity of the older/more distant objects has decreased over time via interactions with the parent cloud, or

2. the velocity at the source has remained at approximately the same value over the last $10^{3}$ years and the distant objects have not been slowed down much by their interaction with the ambient medium.

Large-scale numerical simulations are required to test whether deceleration might be important particularly in the context of the low density environment surrounding a CTTS.

\subsection{Velocity variations along the outflows}

One of the aims of this proper motions study was to investigate how the velocity of $\mathrm{HH}$ objects varies with distance from the source. The pattern of velocity variation was not what we expected however - we have found that the velocity appears to be constant despite the large distances between consecutive HH objects!

Proper motion studies done on the $\sim 3$ pc long HH 34 outflow by Devine et al. (1997) find a systematic decrease in proper motions with distance from the source. This was modelled by Cabrit \& Raga (2000) who investigate whether this observed decrease is due to the ejection velocity at the source increasing over time or that the more distant objects have been slowed down by interactions as the propagate through the parent cloud. They find that the latter scenario is most likely in the case of HH 34.

Our studies however do not show such a decrease in proper motion with distance for the CTTS-driven outflows, in fact they suggest that the velocity has remained approximately constant over at least $10^{3}$ years. As the density of the ambient medium through which the outflow propagates is lower for Class II than Class I sources it is probable that the velocity of the more distant $\mathrm{HH}$ objects has not been greatly affected by its interactions with the parent cloud.

\section{Conclusions}

We have measured the proper motions of a number of $\mathrm{HH}$ objects associated with the CTTS-driven outflows from CW Tau, DG Tau, DO Tau, HV Tau C and RW Aur. The aims of this study were to firstly determine if the driving sources suggested in our original paper (MR04) are correct. Next was to find tangential velocities of the more distant $\mathrm{HH}$ objects in large-scale outflows, which are currently poorly known. Finally, we examine our results to see how tangential velocities evolve with distance from the source.

Our study confirms the previously suggested driving source for some of our objects: HH 826 and HH 827 are driven by CW Tau, HH 702 by DG Tau and HH 835 by RW Aur. However this study also reveals that some of the HH objects previously associated with these CTTSs are not actually driven by them HH 828 and HH 829 are not driven by CW Tau, HH 830 by DG Tau and HH 831 and HH 705 by either DO Tau or HV Tau C. These results are surprising in the case of HH 831 and HH 705 which are well aligned with the "micro-jets" from DO Tau and HV Tau C respectively. While these results show that proper motions are necessary to conclusively determine the source of a $\mathrm{HH}$ object, we contend that examination of the morphology and alignment of newly discovered $\mathrm{HH}$ objects can suggest candidate sources in the first instance, with proper motion studies undertaken at a later date when multi-epoch images are available.

While we now know that these CTTS-driven outflows are not as extended as previously thought in MR04, those from CW Tau, DG Tau and RW Aur still extend to $\sim 0.3 \mathrm{pc}-0.5 \mathrm{pc}$ from the source. The CW Tau and DG Tau outflows are mainly extended in the blue-shifted side, so it is quite likely that the receding red-shifted outflows are obscured. The blue-shifted outflows extend to the edge of the parent cloud (MR04), suggesting that these outflows have blown out. If they are now propagating into a lower density medium it is unlikely that we will observe them.

The tangential velocities determined for the more distant objects in the CW Tau, DG Tau and RW Aur outflows are typically $200 \mathrm{~km} \mathrm{~s}^{-1}$. How velocities of HH objects evolve with distance from the source is an important consideration in parsec-scale outflows. The proper motions of the "micro-jets" and HH objects close to the driving source show that they have a typical velocity of the order of $200 \mathrm{~km} \mathrm{~s}^{-1}$, with very little velocity variation over the lengths of these outflows. This suggests that major outbursts, roughly every thousand years, that give rise to giant $\mathrm{HH}$ complexes could primarily result from additional mass being deposited in an outflow rather than enormous increases in velocity. Further modeling is required to test this hypothesis.

Acknowledgements. D. Froebrich received support from the Cosmo-Grid project, funded by the Program for Research in Third Level Institutions under the National Development Plan and with assistance from the European Regional Development Fund. 


\section{References}

Anglada, G., Rodríguez, L. F., Canto, J., Estalella, R., \& Torrelles, J. M. 1992, ApJ, 395, 494

Andre, P., \& Montmerle, T. 1994, ApJ, 420, 837

Andre, P., Ward-Thompson, D., \& Barsony, M. 1993, ApJ, 406, 122

Andre, P., Ward-Thompson, D., \& Barsony, M. 2000, Protostars and Planets IV, 59

Bacciotti, F., Ray, T. P., Mundt, R., Eislöffel, J., \& Solf, J. 2002, ApJ, 576, 222

Bally, J., \& Devine, D. 1997, Herbig-Haro Flows and the Birth of Stars, IAU Symp., 182, 29

Barsony, M., \& Kenyon, S. J. 1992, ApJ, 384, L53

Bertin, E., \& Arnouts, S. 1996, A\&AS, 117, 393

Bontemps, S., Andre, P., Terebey, S., \& Cabrit, S. 1996, A\&A, 311, 858

Cabrit, S., \& Raga, A. 2000, A\&A, 354, 667

Cabrit, S., Edwards, S., Strom, S. E., \& Strom, K. M. 1990, ApJ, 354, 687

Chini, R., Ward-Thompson, D., Kirk, J. M., et al. 2001, A\&A, 369, 155

Devine, D., Bally, J., Reipurth, B., \& Heathcote, S. 1997, AJ, 114, 2095

Dougados, C., Cabrit, S., Lavalley, C., \& Ménard, F. 2000, A\&A, 357, L61

Duchêne, G., Monin, J.-L., Bouvier, J., \& Ménard, F. 1999, A\&A, 351, 954

Eiroa, C., Miranda, L. F., Anglada, G., Estalella, R., \& Torrelles, J. M. 1994, A\&A, 283, 973

Eislöffel, J., \& Mundt, R. 1992, A\&A, 263, 292

Eislöffel, J., \& Mundt, R. 1998, AJ, 115, 1554

Elias, J. H. 1978, ApJ, 224, 857

Gahm, G. F., Petrov, P. P., Duemmler, R., Gameiro, J. F., \& Lago, M. T. V. T. 1999, A\&A, 352, L95

Gomez, M., Whitney, B. A., \& Kenyon, S. J. 1997, AJ, 114, 1138
Gomez de Castro, A. I. 1993, ApJ, 412, L43

Hamann, F. 1994, ApJS, 93, 485

Hartigan, P., Edwards, S., \& Ghandour, L. 1995, ApJ, 452, 736

Hartigan, P., Morse, J. A., Reipurth, B., Heathcote, S., \& Bally, J. 2001, ApJ, 559, L157

Herbig, G. H. 1977, ApJ, 217, 693

Hirth, G. A., Mundt, R., \& Solf, J. 1994, A\&A, 285, 929

Hirth, G. A., Mundt, R., \& Solf, J. 1997, A\&AS, 126, 437

Hirth, G. A., Mundt, R., Solf, J., \& Ray, T. P. 1994, ApJ, 427, L99

Itoh, Y., Tamura, M., \& Gatley, I. 1996, ApJ, 465, L129

Lada, C. J., \& Wilking, B. A. 1984, ApJ, 287, 610

Lada, C. J. 1987, Star Forming Regions, IAU Symp., 115, 1

Mundt, R., \& Fried, J. W. 1983, ApJ, 274, L83

Mundt, R., \& Eislöffel, J. 1998, AJ, 116, 860

Mundt, R., Brugel, E. W., \& Buehrke, T. 1987, ApJ, 319, 275

McGroarty, F., \& Ray, T. P. 2004, A\&A, 420, 975 MR04

McGroarty, F., Ray, T. P., \& Bally, J. 2004, A\&A, 415, 189

Petrov, P. P., Gahm, G. F., Gameiro, J. F., et al. 2001, A\&A, 369, 993

Ray, T. P. 1987, A\&A, 171, 145

Reipurth, B., \& Bally, J. 2001, ARA\&A, 39, 403

Reipurth, B., Bally, J., \& Devine, D. 1997, AJ, 114, 2708

Reipurth, B., Heathcote, S., Morse, J., Hartigan, P., \& Bally, J. 2002, AJ, 123, 362

Stapelfeldt, K. R., Ménard, F., Watson, A. M., et al. 2003, ApJ, 589, 410

Sun, K., Yang, J., Luo, S., et al. 2003, Chin. J. Astron. Astrophys., 3, 458

Ward-Thompson, D. 2002, Science, 295, 76

Wichmann, R., Bastian, U., Krautter, J., Jankovics, I., \& Rucinski, S. M. 1998, MNRAS, 301, L39 